\title{
Hyperlipidemia and Statins Use for the Risk of New Diagnosed Sarcopenia in Patients with Chronic Kidney: A Population-Based Study
}

\author{
Min-Hua Lin ${ }^{1,2}{ }^{\mathbb{D}}$, She-Yu Chiu ${ }^{1}$, Pei-Hsuan Chang ${ }^{3,4}$, Yu-Liang Lai ${ }^{5,6}$, Pau-Chung Chen ${ }^{7}$ (D) \\ and Wen-Chao Ho ${ }^{4, *}$ \\ 1 Department of Nutrition, China Medical University, Taichung 40402, Taiwan; \\ minhua5356@gmail.com (M.-H.L.); koca345678@gmail.com (S.-Y.C.) \\ 2 Department of Dietetics, Yunlin Christian Hospital, Yunlin 64866, Taiwan \\ 3 Department of Clinical Nutrition, China Medical University Hospital, Taichung 40402, Taiwan; \\ peipei10220312@gmail.com \\ 4 Department of Public Health, China Medical University, Taichung 40402, Taiwan \\ 5 Department of Physical Therapy Medicine and rehabilitation, China Medical University, Hsinchu 302, \\ Taiwan; yuliang22@gmail.com \\ 6 Department of Physical Therapy and School of Medicine, China Medical University, Taichung 40402, Taiwan \\ 7 Department of Public Health, National Taiwan University, Taipei 10617, Taiwan; pchen@ntu.edu.tw \\ * Correspondence: whocmu@gmail.com
}

Received: 7 January 2020; Accepted: 19 February 2020; Published: 26 February 2020

\begin{abstract}
Background: Previous research found that statins, in addition to its efficiency in treating hyperlipidemia, may also incur adverse drug reactions, which mainly include myopathies and abnormalities in liver function. Aim: This study aims to assess the risk for newly onset sarcopenia among patients with chronic kidney disease using statins. Material and Method: In a nationwide retrospective population-based cohort study, 75,637 clinically confirmed cases of chronic kidney disease between 1997 and 2011were selected from the National Health Insurance Research Database of Taiwan. The selection of the chronic kidney disease cohort included a discharge diagnosis with chronic kidney disease or more than 3 outpatient visits with the diagnosis of chronic kidney disease found within 1 year. After consideration of patient exclusions, we finally got a total number of 67,001 cases of chronic kidney disease in the study. The Cox proportional hazards model was used to perform preliminary analysis on the effect of statins usage on the occurrence of newly diagnosed sarcopenia; the Cox proportional hazards model with time-dependent covariates was conducted to take into consideration the individual temporal differences in medication usage, and calculated the hazard ratio (HR) and 95\% confidence interval after controlling for gender, age, income, and urbanization. Results: Our main findings indicated that patients with chronic kidney disease who use statins seem to effectively prevent patients from occurrences of sarcopenia, high dosage of statins seem to show more significant protective effects, and the results are similar over long-term follow-up. In addition, the risk for newly diagnosed sarcopenia among patients with lipophilic statins treatment was lower than that among patients with hydrophilic statins treatment. Conclusion: It seems that patients with chronic kidney disease could receive statin treatment to reduce the occurrence of newly diagnosed sarcopenia. Additionally, a higher dosage of statins could reduce the incidence of newly diagnosed sarcopenia in patients with chronic kidney disease.
\end{abstract}

Keywords: chronic kidney disease; statins; sarcopenia 


\section{Background}

An aging population is becoming an increasingly serious trend around the world. Sarcopenia is a chronic disease that decreases muscle mass and function with increasing age, leading to reduced cardiopulmonary functions, degradation of physiological functions, low quality of life, slow metabolism, risks of falling, dysfunction, death, and increased medical expenses among old people [1-4]. Souza et al. found that patients with sarcopenia are at a higher risk for chronic kidney disease than the general population, and those with chronic kidney disease are more likely to be further suffering from adverse cardiovascular events [5]. In 2002, the Kidney Disease Outcome Quality Initiative (KDOQI) defined chronic kidney disease as "a kidney injury or eGFR lower than $60 \mathrm{~mL} / \mathrm{min} / 1.73 \mathrm{~m}^{2}$, which persists for more than 3 months." Pereira et al. found that the incidence rate for sarcopenia is around $5 \%-9 \%$ in patients in the early stages of chronic kidney disease [6]. Chen et al. found that elderly patients with chronic kidney disease (eGFR $<45 \mathrm{~mL} / \mathrm{min}$ ) are at an increased risk of sarcopenia [7]. In addition, Carrero and Steiber et al. used subjective global assessment to provide evidence that muscle wasting is a predictor of mortality in patients with chronic kidney disease and the severity of muscle wasting is associated with the 5-year survival rate [8,9]. Thus, sarcopenia tends to develop among patients in the later stages of chronic kidney disease and is a significant risk factor. Because of the reduced renal function, patients with chronic kidney disease tend to suffer from several comorbid conditions, including dyslipidemia and related diseases, thus resulting in high risk for cardiovascular diseases. Past literature indicated that moderate- to high-intensity statins should be initiated if patients not receiving dialysis had clinical atherosclerotic cardiovascular disease (ASCVD) such as acute coronary syndromes [10]. For patients with dialysis, guidelines of the 2013 kidney disease: improving global outcomes (KDIGO) and the National Kidney Foundation Kidney Disease Outcomes Quality Initiative (KDOQI) advise that statins treatment should not be administered routinely [11,12], Thus, the probability of being exposed to statins is higher in patients with chronic kidney disease than in the general population. However, recent research indicated that statins may increase patients' risk for myopathies [13-15]. Current literature pertaining to the association between chronic kidney disease (CKD) and prognosis of sarcopenia is sparse and controversial. Moreover, there are six main categories of commonly used statins, with nuances in their mechanisms of action and in their synthesis routes, leading to the difference in pharmacological effects, however, the underlying cause-effect relationship and comorbidities require further investigation.

This study aimed to analyze the medication profile of patients with chronic kidney disease in Taiwan and explore whether statins use is associated with an increased risk for sarcopenia among patients with chronic kidney disease or other consequences. In addition, the study explored the effects of statins dose level and the six main categories of statins on the incidence risk for newly diagnosed sarcopenia to prevent the occurrence of sarcopenia among patients with chronic kidney disease.

\section{Material and Method}

\subsection{Data Source}

The data for the present study were retrieved from the Taiwan National Health Insurance Research Database (NHIRD), which covers $99.9 \%$ of the Taiwanese population of approximately 23 million. The NHIRD is derived from the Taiwanese National Health Insurance (NHI) program, which was launched in 1995 to finance health care for all citizens. The data used in this cohort study were obtained from the Longitudinal National Health Insurance Research Database (LHID) 2000. For the LHID2000, about 1,000,000 representative individuals were randomly sampled from the NHI Registry of Beneficiaries in 2000. LHID2000 contains the following records: the ambulatory care expenditures by visits (CD), details of ambulatory care orders (OO), inpatient expenditures by admissions (DD), details of inpatient orders (DO), expenditures for prescriptions dispensed at contracted pharmacies (GD), registry for beneficiaries (ID). These records are renewed every year. It is one of the largest medical databases in the world, and has been used to analyze the epidemiology in Taiwan extensively [16]. This study was 
approved after a full ethical review by the Institutional Review Board (IRB) of the National Taiwan University Hospital (approval number 20151105), and the IRB waived the need for consent [17]. Those data were accessed anonymously.

\subsection{Definition of Sarcopenia and Selection of ICD Code}

In October 2016, the United States Centers for Disease Control and Prevention (CDC) formally recognized sarcopenia as a disease, encoded as M62.84 in ICD-10-CM. However, the code for sarcopenia is absent in ICD-9-CM, which was used by Taiwan's NHIRD. Therefore, this study followed the suggestion of clinical physicians in practice and referred to the following regulations on code conversion from ICD-9-CM to ICD-10-CM: ICD-9-CM:728.2 can be converted as ICD-10-CM: M62.5 (https://www.icd10data.com/Convert/728.2), and ICD-9-CM: 728.9 can be converted as ICD-10-CM: M62.9 (https://www.icd10data.com/Convert/728.9). In this study, sarcopenia was clinically diagnosed, based on muscle wasting and atrophy (ICD-9-CM: 728.2), and unspecified disorders of muscle, ligament, and fascia (ICD-9-CM: 728.9). This study tracked patients with chronic kidney disease with newly onset sarcopenia, and determined the diagnosis of disease based on the first three digits of the diagnosis codes in the inpatients expenditures by admissions (DD) records for the first records of sarcopenia (ICD-9-CM: $728.2,728.9)$. This study aimed to explore the interaction between the newly onset sarcopenia and other factors. Thus, patients who already showed symptoms of sarcopenia prior to their diagnosis of chronic kidney disease were excluded.

\subsection{Selection of the Chronic Kidney Disease Cohort and Sarcopenia Cases}

This study retrieved data of patients with chronic kidney disease from ambulatory care expenditures by visits (CD) and inpatient expenditures by admissions (DD). Among the 995,347 enrolled patients of the LHID2000 Registry for Beneficiaries of the Taiwan NHIRD, the study reviewed 75,637 clinically confirmed cases of chronic kidney disease between 1997 and 2011. The determination of chronic kidney disease was based on the first three digits of the diagnosis codes in medical records and the International Classification of Disease, Ninth Revision, Clinical Modification (ICD-9-CM) adopted as the selection criteria. Records encoded with ICD-9-CM:250.4,274.1,283.11,403.x1, 404.x2, 404.x3, 440.1, 442.1, 447.3, 572.4, 580-588, 642.1x, 646.2 [18], The selection of the chronic kidney disease cohort included a discharge diagnosis with chronic kidney disease or more than 3 outpatient visits with the diagnosis of chronic kidney disease found within 1 year. We excluded those patients with incomplete records $(\mathrm{N}=1020)$, age smaller than $18(\mathrm{~N}=1946)$, and those who had a diagnosis of sarcopenia prior to January 1, $1997(\mathrm{~N}=73)$. There were 72,598 cases of newly diagnosed patients with chronic kidney disease. Further exclusion criteria were as follows: those with a medical record of sarcopenia before the diagnosis of chronic kidney disease (1922 cases), those who experienced onset of sarcopenia within 1 year of statins administration, and those who were lost in follow-up (3675 cases). In this study, sarcopenia was clinically diagnosed, based on muscle wasting and atrophy (ICD-9-CM: 728.2), and unspecified disorders of muscle, ligament, and fascia (ICD-9-CM: 728.9). Patients who already showed symptoms of sarcopenia prior to their diagnosis of chronic kidney disease were excluded $(\mathrm{N}=1922)$. These exclusions finally resulted in a total number of 67,001 cases of chronic kidney disease, which were used in the study. This study aimed to explore the interaction between the newly onset sarcopenia and medication usage of statins. We tracked patients with chronic kidney disease with newly onset sarcopenia, and determined the diagnosis of disease based on the first three digits of the diagnosis codes in the inpatients expenditures by admissions (DD) record.

\subsection{Assessment of Comorbidities and Confounding Factors}

Diagnostic criteria of comorbidities with sarcopenia in the research population were based on having at least two outpatient records in a year or one inpatient record, and diseases that were included were: hypertension (ICD-9-CM: 401.x-405.x; A-code: A260, A269), hyperlipidemia (ICD-9-CM: 272.x; A-code: A189), atrial fibrillation and flutter (ICD-9-CM:427.3,427.31;A-code:A281), coronary artery 
disease (ICD-9-CM: 410.x-414.x,429.2;A-code:A270, A279), heart failure (ICD-9-CM:428.x;A-code:A289), peripheral artery disease (ICD-9-CM: 250.7, 443.x, 444.2; A-code: A302), diabetes (ICD-9-CM: 250,249; A-code: A181), and liver diseases (ICD-9-CM: 571.2, 571.5-571.7, 572.2-572.5, 572.8, 573.0).

\subsection{Medication Records and Calculation Unit of the National Health Insurance Research Database}

The study's primary drug for analysis is statins, and the six main categories of statins commonly used clinically were included as follows: hydrophilic: pravastatin, fluvastatin, rosuvastatin, and lipophilic: simvastatin, lovastatin, atorvastatin. Medications used by the study subjects before the confirmed diagnosis of sarcopenia included drugs to control hypertension, hyperlipidemia, hyperglycemia, uric acid, gout, and nonsteroidal anti-inflammatory drugs (NSAIDs). According to the 2014 Joint National Committee (JNC8) guidelines for the management of hypertension in adults, the recommended first-line drugs to treat hypertension include diuretics, beta-adrenoceptor antagonists (beta blockers), angiotensin-converting enzyme inhibitors (ACEi), and angiotensin receptor blocker (ARB) [18].

Major drugs for hyperlipidemia management include non-statin drugs to lower blood cholesterol, such as cholestyramine, colestipol, colextran, niceritrol, nicofuranose, acipimox, probucol, and ezetimibe, and fibric acid derivatives (fibrates), such as bezafibrate, clofibrate, etofibrate, fenofibrate, gemfibrozil, and simfibrate. Aspirin is an antiplatelet drug. Drugs used for gout management include primarily xanthine oxidase inhibitors, such as allopurinol and febuxostat, and those used for uric acid management include primarily uricosuric agents, such as benzbromarone, probenecid, and sulfinpyrazone.

\subsection{Exposure to Statins}

The anatomical therapeutic chemical (ATC) classification system of the World Health Organization Collaborating Centre for Drug Statistics Methodology (WHOCC) was used to provide the ATC code [19] for the drugs included into the study's analysis, and the registry for drug prescriptions (DRUG) file from the NHIRD was used to find the pharmacological composition and corresponding drug codes of the related drugs, thus obtaining outpatient and inpatient medication records of the study subjects. The ATC classification system classifies the active ingredients of drugs according to the organ or system on which they act and their therapeutic, pharmacological, and chemical properties, and sets the corresponding defined daily dose (DDD) for each drug. DDD is the standard unit for drugs recommended by the WHO to be used in research to internationally measure drugs. The definition is based on the assumed average daily maintaining dosage used by a 70-kg adult for the drug's primary indication. Exposure of the drugs included in this study were measured and calculated using DDD as the unit, and the following equation was used to calculate the number of DDDs for each prescription of medical usage: Furthermore, in consideration of changes in exposure dosage during the long-term follow-up of personal drugs, cumulative DDDs (cDDDs) were calculated for the follow-up period of each study subject using prescription records of statins medication orders during the follow-up period for each study subject after the onset of chronic kidney disease or until the time of disease onset or the subject was lost to follow-up, and this was used as the drug exposure dose for each study subject.

\subsection{Demographic Data, Including Comorbidity and Socio-Economic Status}

The residence of research subjects was inferred based on the insurance records of the insured and their medical records in the NHIRD. In addition, the degree of urbanization was based on the population density, education level, ratio of population age $\geq 65$ years, development of industries, and distribution of medical resources, which categorized Taiwan into four clusters as follows: "highly urbanized cities and towns," "emerging cities and towns," "regular townships, cities, and districts," and "agricultural and remote townships."

In addition, patterns for smoking and alcohol use of the subjects were are not included in the NHIRD, although these two habits in daily life may seriously influence the results of this research. Therefore, this study used chronic obstructive pulmonary disease (COPD; ICD-9-CM: 491.x, 492.x) 
and alcohol-related diseases (ARD; ICD-9-CM: 291.x, 303.0, 303.9, 305.0, 571.0-571.3) to serve as the alternative variable indicators for chronic smoking and exposure to alcohol use, respectively [20].

\subsection{Data Statistics and Analysis}

\subsubsection{Descriptive Statistics}

The study conducted descriptive statistical analysis according to statins usage by the study subjects on the incidence of sarcopenia, statins dosage used, related risk factors, comorbidity distribution, use of other related drugs, and demographic distribution including gender, age, income, and urbanization level. The Chi-square test was used to analyze differences between the categorical variables, and ANOVA was used to conduct univariate analysis in the differences of the continuous variables. These analyses were performed to infer if there was a difference between the usage of statin drugs regarding the factors mentioned above.

\subsubsection{Analytical Statistics}

In the analysis of the relationship between statins drug usage and sarcopenia incidence in the chronic kidney disease population, the study subjects were categorized into groups according to the statins cDDDs during the follow-up period. Those with cDDDs $<28$ were defined as the non-exposure group, and the exposure groups were categorized into the three groups: low-exposure group, 28-89cDDDs; medium-exposure group, 90-180cDDDs; and high-exposure group, >180cDDDs. This study adopted the Cox proportional hazards model to conduct preliminary analysis on the effect of statins usage on the occurrence of newly diagnosed sarcopenia; further analysis was conducted using the Cox proportional hazards model with time-dependent covariates taking into consideration the individual temporal differences in medication usage, and calculated the hazard ratio (HR) and 95\% confidence interval after controlling for gender, age, income, and urbanization.

\section{Results}

Table 1 shows that 37,215 (55.54\%) patients were male. The mean follow-up time was 6.7 years, mean age of the patients was 60.5 years. Patients $\geq 65$ years had the highest proportion of chronic kidney disease $(44.65 \%)$. In terms of the economics aspect, those with zero monthly income (insured amount) accounted for the highest proportion (32.17\%). For urbanization level of residence, those who resided in second level urbanization areas accounted for the highest proportion (45.61\%). There were 2407 cases of newly diagnosed sarcopenia during the follow-up period in patients with chronic kidney disease. Among all subjects studied, 17,634 (26.3\%) were using statins and 49,367 (73.7\%) were not. Of those who were newly diagnosed with sarcopenia, 547 of them were using statins and 1860 of them were not. Mean follow-up time for those who were using statins and those who were not were 7.5 and 6.3 years, respectively. With respect to economics and urbanization level, those with zero monthly income (insured amount) and those who resided in second level urbanization areas accounted for the highest proportion in both the groups who were using statins and those who were not.

Table 2 shows the comorbidities in patients with chronic kidney disease. Of all the patients, $63.37 \%$ had hypertension, $46.80 \%$ hyperlipidemia, $10.01 \%$ atrial fibrillation and flutter, $21.33 \%$ heart failure, $40.36 \%$ coronary artery disease, $12.04 \%$ peripheral artery disease, $44.15 \%$ diabetes, $6.94 \%$ liver diseases, $23.10 \%$ chronic obstructive pulmonary diseases. All the comorbidities reached statistically significant difference between the group of patients with or without receiving statins.

The cDDDs levels during the follow-up period were analyzed for statins usage. It was found that $6.33 \%, 4.98 \%$, and $15.01 \%$ of those in the low-, medium-, and high-exposure groups $(2889,90,180$, and ( $>180$ cDDDs) used statins, respectively. As for the usage of other drugs related to chronic kidney disease, $34.14 \%$ of patients used aspirin and $61.73 \%$ used NSAIDs. As for drugs other than statins for the management of hyperlipidemia, there were $2.77 \%$ and $12.5 \%$ of patients who used triglyceride lowering drugs and uricosuric agents, respectively. Among them, calcium channel blocker was the 
drug of choice for antihypertensive drugs and $50.06 \%$ patients used it, whereas benzbromarone was the uricosuric agent with the highest proportion (2.19\%). Besides, $1.84 \%$ of patients used drugs for gout management.

Table 1. Demographic characteristics distribution regarding statins use of a chronic kidney disease population in Taiwan.

\begin{tabular}{|c|c|c|c|c|c|c|c|}
\hline \multirow[b]{2}{*}{ Variable } & \multicolumn{2}{|c|}{$\begin{array}{c}\text { All CKD } \\
(N=67,001)\end{array}$} & \multicolumn{2}{|c|}{$\begin{array}{l}\text { Statin User } \\
(\mathrm{N}=17,634)\end{array}$} & \multicolumn{2}{|c|}{$\begin{array}{l}\text { Non-Statin User } \\
\quad(N=49,367)\end{array}$} & \multirow[b]{2}{*}{$p$ Value } \\
\hline & No. & $\%$ & No. & $\%$ & No. & $\%$ & \\
\hline \multicolumn{8}{|l|}{ Sarcopenia } \\
\hline Over all Sarcopenia & 2407 & 3.59 & 547 & 3.10 & 1860 & 3.77 & $<0.0001$ \\
\hline Dialysis & 404 & 0.60 & 84 & 0.48 & 320 & 0.65 & 0.0114 \\
\hline Age of CKD onset ${ }^{\dagger}$ & & & & & & & $<0.0001$ \\
\hline$<45$ & 12,777 & 19.07 & 2157 & 12.23 & 10,620 & 21.51 & \\
\hline $45-65$ & 24,308 & 36.28 & 8550 & 48.49 & 15,758 & 31.92 & \\
\hline$>65$ & 29,916 & 44.65 & 6927 & 39.28 & 22,989 & 46.57 & \\
\hline Med(IQR) & \multicolumn{2}{|c|}{$62.2(48.70-73.50)$} & \multicolumn{2}{|c|}{$61.1(51.70-70.00)$} & \multicolumn{2}{|c|}{$62.9(47.30-74.90)$} & \\
\hline Mean \pm SD & \multicolumn{2}{|c|}{$60.50 \pm 16.70$} & \multicolumn{2}{|c|}{$60.30 \pm 13.00$} & \multicolumn{2}{|c|}{$60.50 \pm 17.80$} & \\
\hline Male sex & 37,215 & 55.54 & 8839 & 50.12 & 28,376 & 57.48 & $<0.0001$ \\
\hline Monthly income ${ }^{\dagger}$, NT\$ & & & & & & & $<0.0001$ \\
\hline 0 & 21,533 & 32.17 & 6593 & 37.39 & 14,960 & 30.30 & \\
\hline $1-15,840$ & 14,152 & 21.12 & 3490 & 19.79 & 10,662 & 21.60 & \\
\hline $15,841-25,000$ & 20,756 & 30.98 & 4915 & 27.87 & 15,841 & 32.09 & \\
\hline$\geqq 25,000$ & 10,540 & 15.73 & 2636 & 14.95 & 7904 & 16.01 & \\
\hline Urbanization level $^{\dagger}$ & & & & & & & $<0.0001$ \\
\hline $\mathrm{I}$ & 16,209 & 24.19 & 4730 & 26.82 & 11,479 & 23.25 & \\
\hline II & 30,560 & 45.61 & 8112 & 46.00 & 22,448 & 45.47 & \\
\hline III & 13,447 & 20.07 & 3265 & 18.52 & 10,182 & 20.63 & \\
\hline IV & 6785 & 10.13 & 1527 & 8.66 & 5258 & 10.65 & \\
\hline \multicolumn{8}{|l|}{ Follow time } \\
\hline $\operatorname{Med}(\mathrm{IQR})$ & \multirow{2}{*}{\multicolumn{2}{|c|}{$\begin{array}{c}6.7(2.70-10.40) \\
6.70 \pm 4.30\end{array}$}} & \multirow{2}{*}{\multicolumn{2}{|c|}{$\begin{array}{c}7.5(4.20-10.70) \\
7.40 \pm 3.90\end{array}$}} & \multicolumn{2}{|c|}{$6.30(2.20-10.20)$} & \\
\hline Mean \pm SD & & & & & 6.40 & 4.40 & \\
\hline
\end{tabular}

CKD, chronic kidney disease; NT\$, New Taiwan dollars; Med (IQR), median (interquartile range); 95\% CI, 95\% confidence interval. Urbanization level: I indicates the highest level of urbanization and IV the lowest. ${ }^{\dagger} \chi^{2}$ test.

Table 2. Clinical characteristics of chronic kidney disease patients in Taiwan.

\begin{tabular}{|c|c|c|c|c|c|c|c|}
\hline \multirow[t]{2}{*}{ Variable } & \multicolumn{2}{|c|}{$\begin{array}{c}\text { All CKD } \\
(\mathrm{N}=67,001)\end{array}$} & \multicolumn{2}{|c|}{$\begin{array}{l}\text { Statin User } \\
(\mathrm{N}=17,634)\end{array}$} & \multicolumn{2}{|c|}{$\begin{array}{l}\text { Non-Statin User } \\
(\mathrm{N}=49,367)\end{array}$} & \multirow[b]{2}{*}{$p$ Value } \\
\hline & No. & $\%$ & No. & $\%$ & No. & $\%$ & \\
\hline \multicolumn{8}{|l|}{ Comorbidity } \\
\hline Hypertension & 42,456 & 63.37 & 15,067 & 85.44 & 27,386 & 55.48 & $<0.0001$ \\
\hline Hyperlipidemia & 31,354 & 46.80 & 15,488 & 87.83 & 15,866 & 32.14 & $<0.0001$ \\
\hline Atrial fibrillation & 6709 & 10.01 & 1933 & 10.96 & 4776 & 9.67 & $<0.0001$ \\
\hline Heart failure & 14,293 & 21.33 & 4808 & 27.27 & 9485 & 19.21 & $<0.0001$ \\
\hline Coronary artery disease & 27,044 & 40.36 & 10,018 & 56.81 & 17,026 & 34.49 & $<0.0001$ \\
\hline Peripheral arterial disease & 8070 & 12.04 & 3406 & 19.31 & 4664 & 9.45 & $<0.0001$ \\
\hline Diabetes disease & 29,584 & 44.15 & 12,261 & 67.53 & 17,323 & 35.09 & $<0.0001$ \\
\hline Liver disease & 4650 & 6.94 & 806 & 4.57 & 3844 & 7.79 & $<0.0001$ \\
\hline COPD & 15,477 & 23.10 & 4469 & 25.34 & 11,008 & 22.3 & $<0.0001$ \\
\hline ARD & 2831 & 4.23 & 553 & 3.14 & 1828 & 3.70 & 0.0005 \\
\hline \multicolumn{8}{|l|}{ Medical medicinet } \\
\hline Statin & & & & & & & $<0.0001$ \\
\hline$<28 \mathrm{cDDDs}$ & 49,367 & 73.68 & 0 & 0.00 & 49,367 & 100.00 & \\
\hline 28-89cDDDs & 4239 & 6.33 & 4239 & 24.04 & 0 & 0.00 & \\
\hline 90-180cDDDs & 3336 & 4.98 & 3336 & 18.92 & 0 & 0.00 & \\
\hline
\end{tabular}


Table 2. Cont.

\begin{tabular}{|c|c|c|c|c|c|c|c|}
\hline \multirow[t]{2}{*}{ Variable } & \multicolumn{2}{|c|}{$\begin{array}{c}\text { All CKD } \\
(\mathrm{N}=67,001)\end{array}$} & \multicolumn{2}{|c|}{$\begin{array}{l}\text { Statin User } \\
(\mathrm{N}=17,634)\end{array}$} & \multicolumn{2}{|c|}{$\begin{array}{l}\text { Non-Statin User } \\
(\mathrm{N}=49,367)\end{array}$} & \multirow[b]{2}{*}{$p$ Value } \\
\hline & No. & $\%$ & No. & $\%$ & No. & $\%$ & \\
\hline$>180 \mathrm{cDDDs}$ & 10,059 & 15.01 & 10,059 & 57.04 & 0 & 0.00 & \\
\hline Statins type & & & & & & & \\
\hline Pravastatin & 2455 & 3.66 & 2455 & 13.92 & 0 & 0.00 & \\
\hline Fluvastatin & 3438 & 5.13 & 3438 & 19.50 & 0 & 0.00 & \\
\hline Atorvastatin & 9090 & 13.57 & 9090 & 51.55 & 0 & 0.00 & \\
\hline Lovastatin & 2932 & 4.38 & 2932 & 16.63 & 0 & 0.00 & \\
\hline Simvastatin & 5192 & 7.75 & 5192 & 29.44 & 0 & 0.00 & \\
\hline Rosuvastatin & 5522 & 8.24 & 5522 & 31.31 & 0 & 0.00 & \\
\hline \multirow[t]{2}{*}{ Variable } & \multicolumn{2}{|c|}{$\begin{array}{c}\text { All CKD } \\
(\mathrm{N}=67,001)\end{array}$} & \multicolumn{2}{|c|}{$\begin{array}{l}\text { Statin User } \\
(N=17,634)\end{array}$} & \multicolumn{2}{|c|}{$\begin{array}{l}\text { Non-Statin User } \\
(\mathrm{N}=49,367)\end{array}$} & \\
\hline & No. & $\%$ & No. & $\%$ & No. & $\%$ & $p$ Value \\
\hline \multicolumn{8}{|l|}{ Medical medicinet } \\
\hline Aspirin & 22,873 & 34.14 & 9957 & 56.46 & 12916 & 26.16 & \\
\hline NSAIDs & 41,357 & 61.73 & 13,131 & 74.46 & 28226 & 57.18 & \\
\hline \multicolumn{8}{|l|}{ Antihypertensive drugs } \\
\hline $\mathrm{ACEi}$ & 21,536 & 32.14 & 8873 & 50.32 & 12663 & 25.65 & $<0.0001$ \\
\hline ARB & 24,839 & 37.07 & 11,391 & 64.60 & 13448 & 27.24 & $<0.0001$ \\
\hline Beta Blocker & 22,283 & 33.26 & 9608 & 54.49 & 12675 & 25.68 & $<0.0001$ \\
\hline $\mathrm{CCB}$ & 33,544 & 50.06 & 12,638 & 71.67 & 20906 & 42.35 & $<0.0001$ \\
\hline Diuretic & 29,650 & 44.25 & 10,624 & 60.25 & 19026 & 38.54 & $<0.0001$ \\
\hline \multicolumn{8}{|l|}{ Anithyperglycemia drugs } \\
\hline Insulins & 14,324 & 21.38 & 5752 & 32.62 & 8572 & 17.36 & $<0.0001$ \\
\hline Biguanides & 16,429 & 24.52 & 8364 & 47.43 & 8065 & 16.34 & $<0.0001$ \\
\hline Sulfonylureas & 19,157 & 28.59 & 9148 & 51.88 & 10009 & 20.27 & $<0.0001$ \\
\hline Thiazolidinedione & 6210 & 9.27 & 4037 & 22.89 & 2173 & 4.40 & $<0.0001$ \\
\hline \multicolumn{8}{|l|}{ Antihyperlipidemic drugs } \\
\hline $\begin{array}{c}\text { Nonstatin lipid-lowering } \\
\text { drug }\end{array}$ & 1854 & 2.77 & 1389 & 7.88 & 465 & 0.94 & $<0.0001$ \\
\hline Fibrate & 8373 & 12.50 & 5344 & 30.31 & 3029 & 6.14 & $<0.0001$ \\
\hline \multicolumn{8}{|l|}{ Antihyperuric acid drugs } \\
\hline Benzbromarone & 1469 & 2.19 & 694 & 3.94 & 775 & 1.57 & $<0.0001$ \\
\hline Probenecid & 7 & 0.01 & 3 & 0.02 & 4 & 0.01 & 0.3893 \\
\hline \multicolumn{8}{|l|}{ Antihyperuric gout drugs } \\
\hline Allopurinol & 1235 & 1.84 & 598 & 3.39 & 637 & 1.29 & $<0.0001$ \\
\hline
\end{tabular}

Abbreviations: $\mathrm{CKD}$, chronic kidney disease; $\mathrm{COPD}$, chronic obstructive pulmonary disease; $\mathrm{ARD}$, alcohol-related disease; ACEi, angiotensin-converting enzyme inhibitor; ARB, angiotensin II receptor blocker; CCB, calcium channel blocker; NSAIDs, nonsteroidal anti-inflammatory drugs. cDDDs, cumulative defined daily doses.

Table 3 displays the risk assessment results for new onset sarcopenia in patients with chronic kidney disease who were using statins versus those who were not, as calculated by the Cox proportional hazard model. The study found the HR for the group that was using statins was 0.709 ( $95 \% \mathrm{CI}, 0.645-0.780$ ). After controlling for age, income, urbanization, comorbidities, and related drug usage, the adjusted HR of new onset sarcopenia for the group that was using statins was $0.753(0.671-0.845)$. For analysis according to the statins cDDDs stratification, HR for new onset sarcopenia for the low-, medium-, and high-exposure groups was 0.909 (0.763-1.069), $1.063(0.895-1.263)$, and $0.547(0.480-0.622)$, respectively. In addition, adjusted HR for the low-, medium-, and high-exposure groups was 0.909 (0.770-1.069), $1.095(0.912-1.313)$, and $0.577(0.498-0.669)$, respectively. The study found that after adjusting for possible influencing factors, the medium-exposure group showed a higher HR, but this did not reach statistical significance, and the high-exposure group showed statistically significant protective effect. 
Table 3. Association between statin and first-time sarcopenia among chronic kidney disease (CKD) patients in Taiwan.

\begin{tabular}{|c|c|c|c|c|}
\hline \multirow{2}{*}{ Variable } & Crude Sarcopenia HR & \multirow{2}{*}{$p$ Value } & Adjust Sarcopenia HR & \multirow{2}{*}{$p$ Value } \\
\hline & $(95 \% \mathrm{CI})$ & & $(95 \% \mathrm{CI})$ & \\
\hline Statin Non-user(<28 cDDDs) & 1 & & 1 & \\
\hline Statin User $(\geq 28 \text { cDDDs })^{*}$ & $0.709(0.645-0.780)$ & $<0.0001$ & $0.753(0.671-0.845)$ & $<0.0001$ \\
\hline \multicolumn{5}{|l|}{ Statin use level * } \\
\hline 28-89cDDDs & $0.909(0.763-1.069)$ & 0.2373 & $0.919(0.770-1.096)$ & 0.3453 \\
\hline 90-180cDDDs & $1.063(0.895-1.263)$ & 0.4843 & $1.095(0.912-1.313)$ & 0.3315 \\
\hline$>180$ cDDDs & $0.547(0.480-0.622)$ & $<0.0001$ & $0.577(0.498-0.669)$ & $<0.0001$ \\
\hline
\end{tabular}

Abbreviations: HR, hazard ratio; $95 \% \mathrm{CI}$, $95 \%$ confidence interval; cDDDs, cumulative defined daily doses. ${ }^{*}$ Statin use and categorization was defined by cumulative prescription (cDDDs) of statin. Models adjusted gender, age, income, urbanization, comorbidity.

Due to further consideration on the difference in time of drug usage between individuals, Cox proportional hazards model was employed with time-dependent covariates and controlling for age, gender, income, urbanization, comorbidities, and other related drug usage to calculate the risk for new onset sarcopenia in patients with chronic kidney disease that were using statins (Table 4). The adjusted HR was 0.963 (0.835-1.109), 1.241 (1.043-1.478), $0.771(0.598-0.994)$, and $0.638(0.453-0.899)$ for those using statins, those in the low-, medium-, and high-exposure groups, respectively. The results showed a significant dose-responsive trend.

Table 4. Association between statin and first-time sarcopenia among CKD patients in Taiwan with time- dependent covariates for statin.

\begin{tabular}{|c|c|c|c|c|}
\hline \multirow{2}{*}{ Variable } & Crude Sarcopenia HR & \multirow{2}{*}{$p$ Value } & Adjust Sarcopenia HR & \multirow{2}{*}{$p$ Value } \\
\hline & $(95 \% \mathrm{CI})$ & & $(95 \% \mathrm{CI})$ & \\
\hline Statin Non-User (<28 cDDDs) & 1 & & 1 & \\
\hline Statin User $(\geq 28 \mathrm{cDDDs}) *$ & $0.845(0.741-0.965)$ & 0.0129 & $0.963(0.835-1.109)$ & 0.5987 \\
\hline Statin use level * & & $<0.0001$ & & 0.0004 \\
\hline $28-89 \mathrm{cDDDs}$ & $1.113(0.941-1.316)$ & & $1.241(1.043-1.478)$ & \\
\hline 90-180cDDDs & $0.675(0.526-0.865)$ & & $0.771(0.598-0.994)$ & \\
\hline$>180$ cDDDs & $0.543(0.387-0.762)$ & & $0.638(0.453-0.899)$ & \\
\hline
\end{tabular}

Abbreviations: HR, hazard ratio; $95 \%$ CI, $95 \%$ confidence interval; cDDDs, cumulative defined daily doses. ${ }^{*}$ Statin use and categorization was defined by cumulative prescription (cDDDs) of statin. Models adjusted gender, age, income, urbanization, comorbidity.

The Kaplan-Meier method was used to estimate cumulative incidence of new onset sarcopenia during the follow-up period. The cumulative incidence of new onset sarcopenia during the follow-up period for the group that was using statins was lower than that for the group that was not using statins (Figure 1). Additionally, in terms of the follow-up period, the cumulative incidence rate of new onset sarcopenia for the group that was using statins gradually approached the cumulative incidence rate for the group that was not using statins, with significant difference $(p<0.0001)$ using the log-rank test.

Statins can be divided by their pharmacological characteristics into the two main categories as hydrophilic and lipophilic. The adjusted HR of new onset sarcopenia in patients with chronic kidney disease who were using hydrophilic statins in comparison to those who were not using statins was 1.013 (95\% CI, 0.826-1.241; Table 5). The cDDDs stratification results showed that for those who were using hydrophilic statins, the adjusted HR showed decreased incidence risk for sarcopenia in the medium- and high-exposure groups, with a significant dose-responsive trend $(p=0.0283)$. The adjusted HR of new onset sarcopenia in patients with chronic kidney disease who were using lipophilic statins in comparison to those who were not using statins was 0.912 (95\% CI, 0.763-1.090; Table 6). The cDDDs stratification results showed that, for those who were using lipophilic statins, the adjusted HR decreased the incidence risk for sarcopenia in the medium- and high-exposure 
groups, with a significant dose-responsive trend $(p=0.0066)$. Table 7 shows the risk assessment results comparing sarcopenia incidence for those who were using lipophilic and hydrophilic statins. The results showed that regardless of dosage stratification or correction for influencing factors, the risk for sarcopenia incidence was lower for those who were using lipophilic statins than for those who were using hydrophilic statins with no significant difference. Nonetheless, both lipophilic and hydrophilic statins displayed a protective trend.

\section{Kaplan-Meier curves}

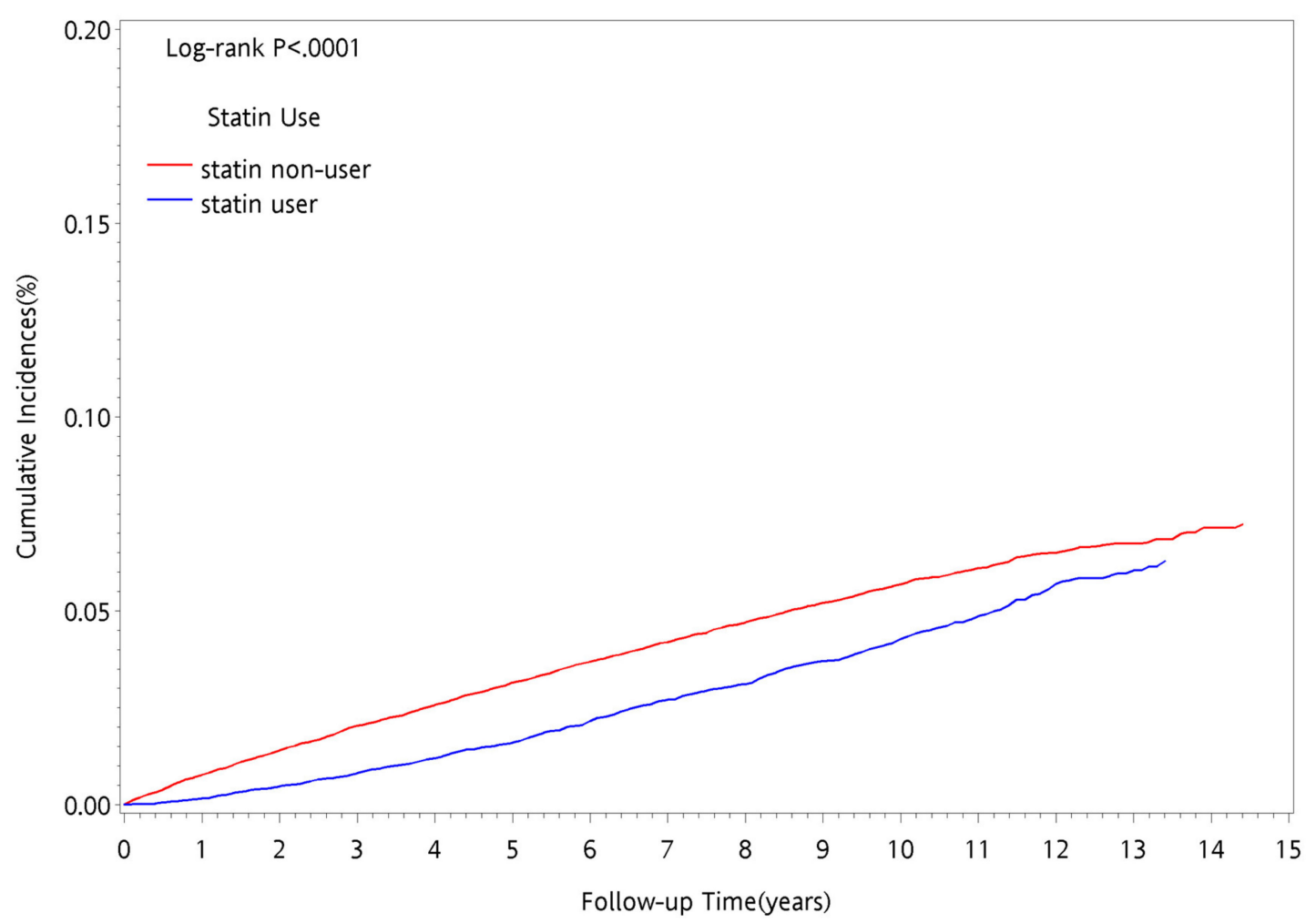

Figure 1. Cumulative incidence of sarcopenia by statins use among CKD patients in Taiwan during follow time.

Table 5. Association between hydrophilic of statins and first-time sarcopenia among CKD patients in Taiwan.

\begin{tabular}{|c|c|c|c|c|}
\hline Variable & $\begin{array}{c}\text { Crude Sarcopenia HR } \\
(95 \% \text { CI })\end{array}$ & $p$ Trend & $\begin{array}{c}\text { Adjust Sarcopenia HR } \\
(95 \% \text { CI })\end{array}$ & $p$ Value \\
\hline Statin Non-user (<28 cDDDs) & 1 & & 1 & \\
\hline Hydrophilic Statin User & $0.895(0.735-1.090)$ & 0.2706 & $1.013(0.826-1.241)$ & 0.9046 \\
\hline Hydrophilic Statin use level * & & 0.015 & & 0.0283 \\
\hline 28-89cDDDs & $1.275(0.960-1.692)$ & & $1.404(1.053-1.870)$ & \\
\hline 90-180cDDDs & $0.827(0.583-1.720)$ & & $0.933(0.655-1.327)$ & \\
\hline$>180 \mathrm{cDDDs}$ & $0.589(0.391-0.889)$ & & $0.684(0.452-1.036)$ & \\
\hline
\end{tabular}

* Statin use and categorization was defined by cumulative prescription (cDDDs) of statin. Models adjusted gender, age, income, urbanization, comorbidity. 
Table 6. Association between lipophilic of statins and first-time sarcopenia among CKD patients in Taiwan.

\begin{tabular}{|c|c|c|c|c|}
\hline Variable & $\begin{array}{c}\text { Crude Sarcopenia HR } \\
(95 \% \text { CI })\end{array}$ & $p$ Trend & $\begin{array}{c}\text { Adjust Sarcopenia HR } \\
(95 \% \text { CI) }\end{array}$ & $p$ Trend \\
\hline Statin Non-user (<28 cDDDs) & 1 & & 1 & \\
\hline Lipophilic Statin User & $0.812(0.685-0.964)$ & 0.0172 & $0.912(0.763-1.090)$ & 0.3121 \\
\hline Lipophilic Statin use level * & & 0.0009 & & 0.0066 \\
\hline 28-89cDDDs & $1.046(0.853-1.282)$ & & $1.157(0.938-1.426)$ & \\
\hline 90-180cDDDs & $0.571(0.403-0.809)$ & & $0.646(0.454-0.919)$ & \\
\hline$>180 \mathrm{cDDDs}$ & $0.468(0.259-0.846)$ & & $0.543(0.299-0.984)$ & \\
\hline
\end{tabular}

Abbreviations: HR, hazard ratio; 95\% CI, 95\% confidence interval; cDDDs, cumulative defined daily doses. * Statin use and categorization was defined by cumulative prescription (cDDDs) of statin. Models adjusted gender, age, income, urbanization, comorbidity.

Table 7. Association between lipophilic and hydrophilic of statins and first-time sarcopenia among CKD patients in Taiwan.

\begin{tabular}{rcccc}
\hline \multicolumn{1}{c}{ Variable } & $\begin{array}{c}\text { Crude Sarcopenia HR } \\
(\mathbf{9 5 \%} \text { CI) }\end{array}$ & $\boldsymbol{p}$ Trend & $\begin{array}{c}\text { Adjust Sarcopenia HR } \\
(\mathbf{9 5 \%} \text { CI) }\end{array}$ & $p$ Trend \\
\hline $\begin{array}{l}\text { Hydrophilic User } \\
\text { Lipophilic Statin User }\end{array}$ & $\mathbf{1}$ & & $\mathbf{1}$ & \\
Lipophilic Statin use level * & $0.919(0.712-1.185)$ & 0.5136 & $0.933(0.723-1.205)$ & 0.5961 \\
28-89cDDDs & $0.825(0.585-1.165)$ & 0.2752 & $0.856(0.606-1.210)$ & 0.3789 \\
90-180cDDDs & $0.685(0.419-1.120)$ & 0.1317 & $0.698(0.426-1.144)$ & 0.1542 \\
$>180$ cDDDs & $0.842(0.410-1.729)$ & 0.6394 & $0.866(0.419-1.786)$ & 0.6961 \\
\hline
\end{tabular}

Abbreviations: HR, hazard ratio; 95\% CI, 95\% confidence interval; cDDDs, cumulative defined daily doses. * Statin use and categorization was defined by cumulative prescription (cDDDs) of statin. Models adjusted gender, age, income, urbanization, comorbidity.

Commonly used statins can be further subdivided into six primary categories with different therapeutic and harm effects due to slight differences in their pharmacodynamics effect, metabolic pathway, and pharmacological mechanism. Table 8 shows the comparison of risk assessment results of new onset sarcopenia for those who were using the six types of statins and for those who were not using statins. The results found that before adjustment, all types of statin drugs presented a decreased incidence risk for sarcopenia. After controlling for age, gender, income, urbanization, comorbidities, and related drug usage, the adjusted HR for fluvastatin was 0.883 (95\% CI, 0.733-1.064) and for lovastatin was $0.924(0.762-1.121)$. With the exception of the two drugs mentioned above which did not reach statistically significant differences, the remaining statin drugs all showed significantly decreased risk for incidence of sarcopenia. In addition, usage of any of the six types of statins all showed a decreasing trend for HR of sarcopenia incidence when compared to those who were not using statins.

Sensitivity analysis results for the effects of statin on newly onset sarcopenia among CKD patients in Taiwan with time-dependent covariates are shown in Table 9. Past literature has shown that gender, age, menopausal women, dialysis, and hyperlipidemia may be important risk factors for sarcopenia. The sensitivity analysis results showed that statistically significant differences were found in men $(p=0.0016)$ and in the age stratification group younger than 65 . There was no significance difference of the risk of sarcopenia caused by the use of statins in women before and after menopause. There is a statistically significant difference in the risk of sarcopenia caused by the use of statins in patients with hyperlipidemia $(p=0.0007$, which shows that the higher the dose, the more significant the protective effect. Because there were only 404 dialysis patients and there were only few patients with dialysis who were diagnosed sarcopenia with usage of statins, so they were not statistically analyzed. In addition, when the diagnosis department of sarcopenia was used in stratification, the results showed that the risk of sarcopenia caused by the use of statin drugs in non-home medicine diagnosis was statistically significant $(p=0.0011)$. However, whether or not it is diagnosed in a family medicine department, 
the risk of sarcopenia caused by the use of statins has a dose-response effect. The higher the dose, the better the protective effect.

Table 8. Association between six types of statins and first-time sarcopenia among CKD patients in Taiwan.

\begin{tabular}{|c|c|c|c|c|}
\hline \multirow{2}{*}{ Variable } & Sarcopenia HR & \multirow{2}{*}{$p$ Value } & Adjust Sarcopenia HR & \multirow{2}{*}{$p$ Value } \\
\hline & $(95 \% \mathrm{CI})$ & & $(95 \% \mathrm{CI})$ & \\
\hline Statin Non-Use & 1 & & 1 & \\
\hline Statin use & $0.709(0.645-0.780)$ & $<0.0001$ & $0.753(0.671-0.845)$ & $<0.0001$ \\
\hline \multicolumn{5}{|l|}{ Hydrophilic Statin } \\
\hline Pravastatin & $0.681(0.542-0.855)$ & 0.0010 & $0.766(0.607-0.968)$ & 0.0257 \\
\hline Fluvastatin & $0.785(0.655-0.940)$ & 0.0086 & $0.883(0.733-1.064)$ & 0.1917 \\
\hline Rosuvastatin & $0.407(0.330-0.501)$ & $<0.0001$ & $0.448(0.362-0.555)$ & $<0.0001$ \\
\hline \multicolumn{5}{|l|}{ Lipophilic Statin } \\
\hline Lovastatin & $0.831(0.690-1.001$ & 0.0508 & $0.924(0.762-1.121)$ & 0.4238 \\
\hline Simvastatin & $0.775(0.667-0.902)$ & 0.0010 & $0.850(0.724-0.997)$ & 0.0452 \\
\hline Atorvastatin & $0.702(0.620-0.795)$ & $<0.0001$ & $0.791(0.691-0.906)$ & 0.0007 \\
\hline
\end{tabular}

Abbreviations: HR, hazard ratio; 95\% CI, 95\% confidence interval. Models adjusted gender, age, income, urbanization, comorbidity.

Table 9. Sensitivity analysis results.

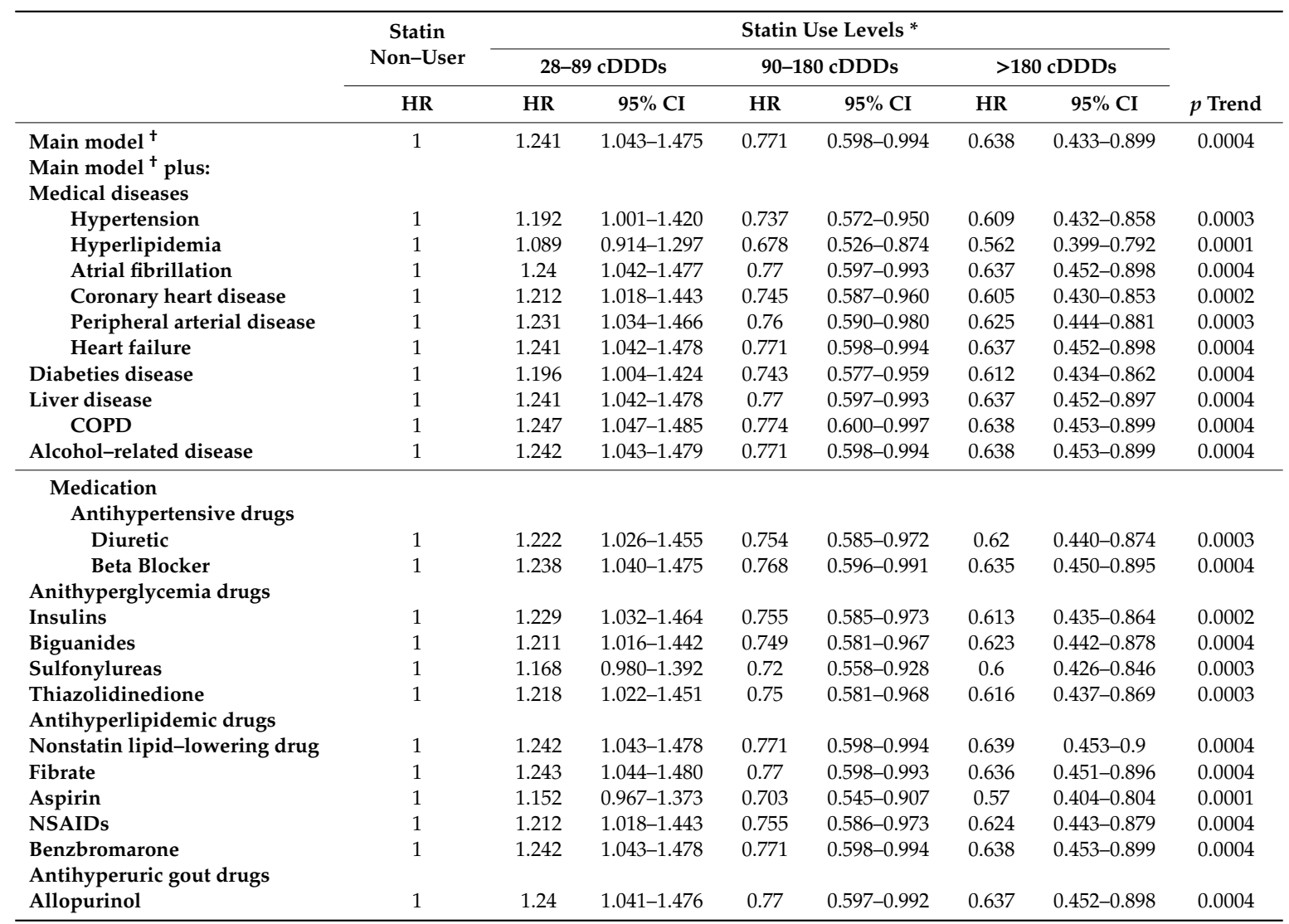


Table 9. Cont.

\begin{tabular}{|c|c|c|c|c|c|c|c|c|}
\hline & \multirow{3}{*}{$\begin{array}{c}\begin{array}{c}\text { Statin } \\
\text { Non-User }\end{array} \\
\text { HR }\end{array}$} & \multicolumn{6}{|c|}{ Statin Use Levels * } & \multirow[b]{3}{*}{$p$ Trend } \\
\hline & & \multicolumn{2}{|c|}{ 28-89 cDDDs } & \multicolumn{2}{|c|}{ 90-180 cDDDs } & \multicolumn{2}{|c|}{$>180$ cDDDs } & \\
\hline & & HR & $95 \%$ CI & HR & $95 \%$ CI & HR & $95 \%$ CI & \\
\hline \multicolumn{9}{|c|}{ Subgroup analysist } \\
\hline Female & 1 & 1.163 & $0.917-1.476$ & 0.88 & $0.641-1.208$ & 0.699 & $0.451-1.084$ & 0.1596 \\
\hline Male & 1 & 1.364 & $1.055-1.762$ & 0.63 & $0.411-0.968$ & 0.568 & $0.327-0.986$ & 0.0016 \\
\hline$\geq 65$ & 1 & 1.329 & $1.023-1.727$ & 1.003 & $0.703-1.430$ & 0.667 & $0.391-1.136$ & 0.0657 \\
\hline \multicolumn{9}{|c|}{ Menopause } \\
\hline Yes & 1 & 1.226 & $0.951-1.582$ & 0.903 & $0.641-1.272$ & 0.649 & $0.393-1.072$ & 0.1004 \\
\hline No & 1 & 0.744 & $0.375-1.478$ & 0.698 & $0.305-1.600$ & 0.85 & $0.343-2.109$ & 0.7089 \\
\hline \multicolumn{9}{|c|}{ Hyperlipidemia } \\
\hline No & 1 & 1.224 & $1.014-1.496$ & 0.716 & $0.541-0.948$ & 0.604 & $0.463-0.951$ & 0.0011 \\
\hline
\end{tabular}

Abbreviations: cDDDs, cumulative defined daily doses; HR, hazard ratio; 95\% CI, 95\% confidence interval; COPD, chronic obstructive pulmonary disease; ACEi, angiotensin-converting enzyme inhibitor; ARB, Angiotensin II Receptor Blocker; Alpha-Gi, alpha-glucosidase inhibitor; NSAIDs, nonsteroidal anti-inflammatory drugs. * Statin use and categorization was defined by cumulative prescription (cDDDs) of statin. ${ }^{\dagger}$ Models adjusted gender, age, income, urbanization, comorbidity.

\section{Discussion}

\subsection{Investigation on Statins Usage and Sarcopenia}

Previous research have pointed out that patients with chronic kidney disease often suffer from metabolic acidosis, which may lead to increase in protein catabolism, decrease in protein synthesis, insulin resistance, chronic inflammation, decrease in serum leptin concentration that may cause increase in the breakdown of branched amino acids and muscle proteins, thus inhibiting muscle protein synthesis [21-24] and resulting in increased occurrences of sarcopenia in late stage patients with chronic kidney disease [25]. However, there has been no research related to the exploration on the usage of statins in patients with chronic kidney disease and the incidence risk of sarcopenia. The results of this study showed that patients with chronic kidney disease who use statins can effectively decrease the incidence risk of sarcopenia, and the protective effect is most significant for those who use high dosage statins. Possible reasoning for this could be that chronic kidney patients may have higher risk for cardiovascular diseases, diabetes, and cerebral stroke. During the follow-up period of this study, usage of statins can prevent mortality from cardiovascular diseases in this particular population of patients and may even reduce the rate of Glomerular filtration rate GFR reduction thus decreasing kidney functional injury [26-28]. Furthermore, it could decrease the deterioration of proteinuria [26], thus delaying the starting time for dialysis treatment [29] and indirectly increasing muscle protein preservation. Therefore, there is a protective effect in statins usage for patients with chronic kidney disease regarding sarcopenia incidence risk. Furthermore, weight loss and changing life habits were also one of the important goals in addition to medication treatment in the 2015 Taiwan Chronic Kidney Disease Clinical Guidelines [30]. Therefore, change in life habits, increase in activity, and weight reduction may influence statins effects on the risk of newly onset sarcopenia.

\subsection{Investigation on Related Factors of the Six Types of Statins and Sarcopenia}

Sarcopenia is the combination of age-related progressive muscle bulk reduction and the decrease in muscle function and body performance [31]. Mikus et al. collected 37 patients between the age of 25-59 years who had metabolic syndromes and randomly assigned the patients to the two groups of aerobic exercise or aerobic exercise plus simvastatin administration (40 mg each day) [32]. The experiment time was 12 weeks and results showed that the group that underwent exercise with simvastatin administration 
had a $4.5 \%(p<0.05)$ decrease in citrate synthase activity in skeletal muscles; this showed that although simvastatin can increase cardiopulmonary health and reduce abnormal blood lipid concentration, it can also cause skeletal muscle injury. Panza conducted three different studies on three different populations to evaluate how statins induce muscle power changes in patients with myopathy [33]. The first study randomly assigned atorvastatin $(80 \mathrm{mg}$ ) or a placebo to healthy individuals and assessed the effects on muscle function; the second study administered simvastatin + placebo or simvastatin + coenzyme $Q$ to patients who suffered from muscle soreness and evaluated the muscle power and symptoms before and after treatment; the third study provided different types of drugs, including rosuvastatin, simvastatin, pravastatin, in different dosages to clinical patients who were experiencing muscle pain. The results for the first study showed that subjects who used atorvastatin had a decrease in muscles, but the phenomenon was also observed in the placebo group, thus showing that the change in muscle power was not specific to the usage of atorvastatin. In the second study with the randomized cross-experiment using simvastatin and placebo, it was shown that the subjects who used simvastatin did not show any decrease in muscle power. For the third study, it was shown that different types of statins had different effects regarding risk for decrease in muscle power. For all three studies, the observed change in muscle power all showed no statistical significant, thus displayed that in the short term, the adverse effect of statins is unrelated to functional changes and different types of statins have different effects on muscle power and muscle function, displaying a possible difference in mechanism for the risk to cause sarcopenia.

This study performed stratified analysis in the six types of statins, and the results, after controlling for influencing factors, showed no statistical significant differences for fluvastatin and lovastatin. However, all six types of statins showed a protective effect regarding new onset sarcopenia. In addition, the study found that lipophilic statins had lower incidence risk for the onset of sarcopenia than hydrophilic statins, and this did not reach a statistical significant difference. Thus, this topic requires further exploration in the future.

\section{Conclusions}

Our main findings of this retrospective and large-scaled cohort study indicated that patients with chronic kidney disease who use statins seem to effectively prevent patients from occurrences of sarcopenia, high dosage of statins seem to show more significant protective effects, and the results are similar over long-term follow-up. In addition, the study observed that usage of hydrophilic statins has a higher risk for newly onset sarcopenia than the use of lipophilic statins. However, the study was limited to a Taiwanese population, the findings and conclusions thus may not be generalized to other populations.

Patients with chronic kidney disease are a susceptible population, and furthermore, a high risk population for sarcopenia, which could significantly affect chances for comorbidities such as cardiovascular diseases and increase mortality risk. Therefore, special protective measures should be provided for patients with chronic kidney disease to avoid comorbid harm from sarcopenia that may cause risks such as loss of muscle mass, decrease in muscle power, decrease in walking speed, and decrease in daily activity function. The results of this study showed that patients with chronic kidney disease given statins treatment showed effects regarding risk for newly onset sarcopenia. In the future, the usage of statins in patients with chronic kidney disease could be considered to decrease the incidence of newly diagnosed sarcopenia.

\subsection{Research Advantages}

1. This study used the Longitudinal Health Insurance Database from the 2000 Registry for Beneficiaries of the Taiwan NHIRD, which is a large study sample size with good representativeness and low possibility for selection bias.

2. The NHIRD contains actual medical record of patients, thus decreasing recall bias.

3. The study subjects were selected based on disease diagnosis codes, and the disease diagnosis and coding were all processed by physicians, thus decreasing the occurrence of misclassification. 
4. The follow-up time of this study is 15 years, and provides results and trends on the incidence risk for sarcopenia in patients with chronic kidney disease who use statins on the long-term.

\subsection{Research Limitations}

First of all, important influencing factors, such as laboratory data (serum and biochemistry examination), life habits (smoking and alcohol), family history (hereditary diseases), and surrounding living environment quality (exposure to pollution), could not be obtained from the NHIRD, and thus could not be corrected. Second, this study presumed that all study subjects took the prescribed statins medication according to medical order. However, there is still a possibility that some of the subjects may not have adhered to the medical orders and did not take the medication, thus the study had overestimated the actual statins dosage used by the study subjects. Third, the NHIRD did not include detailed information on laboratory data. For example, we were unable to estimate glomerular filtration rate or detect proteinuria at baseline and could not adjust for these unmeasured potential confounders. Additionally, actual examination values of muscle power, walking speed, and skeletal muscle mass of the newly onset sarcopenia subjects could not be known.

Author Contributions: Conceptualization, M.-H.L., W.-C.H.; Methodology, M.-H.L., W.-C.H. and Y.-L.L.; Formal Analysis, S.-Y.C. and P.-H.C.; Data Curation, M.-H.L. and P.-H.C.; Writing-Original Draft Preparation, M.-H.L., P.-H.C. and Y.-L.L.; Writing-Review \& Editing, M.-H.L., W.-C.H. and P.-C.C.; Supervision, W.-C.H. and P.-C.C. All authors have read and agreed to the published version of the manuscript.

Funding: This research received no external funding.

Acknowledgments: We appreciate the support of the Taiwan Food and Drug Administration and China Medical University.

Conflicts of Interest: The authors declare no conflicts of interest.

\section{References}

1. Delmonico, M.J.; Harris, T.B.; Visser, M.; Park, S.W.; Conroy, M.B.; Velasquez-Mieyer, P.; Boudreau, R.; Manini, T.M.; Nevitt, M.; Newman, A.B.; et al. Longitudinal study of muscle strength, quality, and adipose tissue infiltration. Am. J. Clin. Nutr. 2009, 90, 1579-1585. [PubMed]

2. Goodpaster, B.H.; Park, S.W.; Harris, T.B.; Kritchevsky, S.B.; Nevitt, M.; Schwartz, A.V.; Simonsick, E.M.; Tylavsky, F.A.; Visser, M.; Newman, A.B.; et al. The loss of skeletal muscle strength, mass, and quality in older adults: The health, aging and body composition study. J. Gerontol. A Biol. Sci. Med. Sci. 2006, 61, 1059-1064. [CrossRef] [PubMed]

3. Lim, S.; Kwon, S.Y.; Yoon, J.W.; Kim, S.Y.; Choi, S.H.; Park, Y.J.; Yoon, H.I.; Chang, Y.S.; Lee, J.H.; Lee, C.T.; et al. Association between Body Composition and Pulmonary Function in Elderly People: The Korean Longitudinal Study on Health and Aging. Obesity 2011, 19, 631-638. [CrossRef] [PubMed]

4. Woo, J.; Leung, J.; Sham, A.; Kwok, T. Defining sarcopenia in terms of risk of physical limitations: A 5-year follow-up study of 3153 Chinese men and women. J. Am. Geriatr. Soc. 2009, 57, 2224-2231. [CrossRef] [PubMed]

5. Harada, K.; Suzuki, S.; Ishii, H.; Aoki, T.; Hirayama, K.; Shibata, Y.; Negishi, Y.; Sumi, T.; Kawashima, K.; Kunimura, A.; et al. Impact of Skeletal Muscle Mass on Long-Term Adverse Cardiovascular Outcomes in Patients With Chronic Kidney Disease. Am. J. Cardiol. 2017, 119, 1275-1280. [CrossRef] [PubMed]

6. Pereira, R.A.; Cordeiro, A.C.; Avesani, C.M.; Carrero, J.J.; Lindholm, B.; Amparo, F.C.; Amodeo, C.; Cuppari, L.; Kamimura, M.A. Sarcopenia in chronic kidney disease on conservative therapy: Prevalence and association with mortality. Nephrol. Dial. Transplant. 2015, 30, 1718-1725. [CrossRef]

7. Chen, L.K.; Lee, W.J.; Peng, L.N.; Liu, L.K.; Arai, H.; Akishita, M.; Asian Working Group for Sarcopenia. Recent Advances in Sarcopenia Research in Asia: 2016 Update from the Asian Working Group for Sarcopenia. J. Am. Med. Dir. Assoc. 2016, 17, 767. [CrossRef]

8. Carrero, J.J.; Chmielewski, M.; Axelsson, J.; Snaedal, S.; Heimbürger, O.; Bárány, P.; Suliman, M.E.; Lindholm, B.; Stenvinkel, P.; Qureshi, A.R. Muscle atrophy, inflammation and clinical outcome in incident and prevalent dialysis patients. Clin. Nutr. (Edinb. Scotl.) 2008, 27, 557-564. [CrossRef] 
9. Steiber, A.; Leon, J.B.; Secker, D.; McCarthy, M.; McCann, L.; Serra, M.; Sehgal, A.R.; Kalantar-Zadeh, K. Multicenter study of the validity and reliability of subjective global assessment in the hemodialysis population. J. Ren. Nutr. 2007, 17, 336-342. [CrossRef]

10. Stone, N.J.; Robinson, J.G.; Lichtenstein, A.H.; Bairey Merz, C.N.; Blum, C.B.; Eckel, R.H.; Goldberg, A.C.; Gordon, D.; Levy, D.; Lloyd-Jones, D.M.; et al. American College of Cardiology/American Heart Association Task Force on Practice Guidelines. 2013 ACC/AHA guideline on the treatment of blood cholesterol to reduce atherosclerotic cardiovascular risk in adults: A report of the American College of Cardiology/American Heart Association Task Force on Practice Guidelines. Circulation 2014, 129, 1-45.

11. Wanner, C.; Tonelli, M. KDIGO Clinical Practice Guideline for Lipid Management in CKD: Summary of recommendation statements and clinical approach to the patient. Kidney Int. 2014, 85, 1303-1309. [CrossRef] [PubMed]

12. Sarnak, M.J.; Bloom, R.; Muntner, P.; Rahman, M.; Saland, J.M.; Wilson, P.W.; Fried, L. KDOQI US commentary on the 2013 KDIGO Clinical Practice Guideline for Lipid Management in CKD. Am. J. Kidney Dis. 2015, 65, 354-366. [CrossRef] [PubMed]

13. Gillett, R.C., Jr.; Norrell, A. Considerations for safe use of statins: Liver enzyme abnormalities and muscle toxicity. Am. Fam. Physician. 2011, 83, 711-716. [PubMed]

14. Visser, M.; Kritchevsky, S.B.; Newman, A.B.; Goodpaster, B.H.; Tylavsky, F.A.; Nevitt, M.C.; Harris, T.B. Lower serum albumin concentration and change in muscle mass: The Health, Aging and Body Composition Study. Am. J. Clinic. Nutrit. 2005, 82, 531-537. [CrossRef]

15. Scott, D.; Blizzard, L.; Fell, L.; Jones, G. Statin therapy, muscle function and falls risk in community-dwelling older adults. Qjm-Int. J. Med. 2009, 102, 625-633. [CrossRef]

16. Hsing, A.W.; Ioannidis, J.P. Nationwide Population Science: Lessons from the Taiwan National Health Insurance Research Database. JAMA Intern. Med. 2015, 175, 1527-1529. [CrossRef]

17. National Health Insurance Research Database, Taiwan. Available online: http://nhird.nhri.org.tw/en/index. htm (accessed on 9 January 2020).

18. James, P.A.; Oparil, S.; Carter, B.L.; Cushman, W.C.; Dennison-Himmelfarb, C.; Handler, J.; Lackland, D.T.; LeFevre, M.L.; MacKenzie, T.D.; Ogedegbe, O.; et al. 2014 evidence-based guideline for the management of high blood pressure in adults: Report from the panel members appointed to the Eighth Joint National Committee (JNC 8). JAMA 2014, 311, 507-520. [CrossRef]

19. WHO. Guidelines for ATC Classification and DDD Assignment 2016; WHO: Geneva, Switzerland, 2016.

20. Tsan, Y.T.; Lee, C.H.; Ho, W.C.; Lin, M.H.; Wang, J.D.; Chen, P.C. Statins and the risk of hepatocellular carcinoma in patients with hepatitis C virus infection. J. Clin. Oncol. 2013, 31, 1514-1521. [CrossRef]

21. Mehrotra, R.; Kopple, J.D.; Wolfson, M. Metabolic acidosis in maintenance dialysis patients: Clinical considerations. Kidney Int. Suppl. 2003, 64, 13-25. [CrossRef]

22. Lim, V.S.; Yarasheski, K.E.; Flanigan, M.J. The effect of uraemia, acidosis, and dialysis treatment on protein metabolism: A longitudinal leucine kinetic study. Nephrol. Dial. Transplant. 1998, 13, 1723-1730. [CrossRef]

23. Graham, K.A.; Reaich, D.; Channon, S.M.; Downie, S.; Goodship, T.H. Correction of acidosis in hemodialysis decreases whole-body protein degradation. J. Am. Soc. Nephrol. 1997, 8, 632-637.

24. Reaich, D.; Channon, S.M.; Scrimgeour, C.M.; Daley, S.E.; Wilkinson, R.; Goodship, T.H. Correction of acidosis in humans with CRF decreases protein degradation and amino acid oxidation. Am. J. Physiol. 1993, 265, E230-E235. [CrossRef]

25. Souza, V.A.; Oliveira, D.; Barbosa, S.R.; Corrêa, J.O.D.A.; Colugnati, F.A.B.; Mansur, H.N.; Fernandes, N.M.D.S.; Bastos, M.G. Sarcopenia in patients with chronic kidney disease not yet on dialysis: Analysis of the prevalence and associated factors. PLoS ONE 2017, 12, e0176230. [CrossRef]

26. Bianchi, S.; Bigazzi, R.; Caiazza, A.; Campese, V.M. A controlled, prospective study of the effects of atorvastatin on proteinuria and progression of kidney disease. Am. J. Kidney Dis. 2003, 41, 565-570. [CrossRef] [PubMed]

27. Fielding, C.J.; Fielding, P.E. Cellular cholesterol efflux. Biochim. Biophys. Acta. 2001, 1533, 175-189. [CrossRef]

28. Shepherd, J.; Kastelein, J.J.; Bittner, V.; Deedwania, P.; Breazna, A.; Dobson, S.; Wilson, D.J.; Zuckerman, A.; Wenger, N.K.; Treating to New Targets Investigators. Effect of intensive lipid lowering with atorvastatin on renal function in patients with coronary heart disease: The Treating to New Targets (TNT) study. Clin. J. Am. Soc. Nephrol. 2007, 2, 1131-1139. [CrossRef] [PubMed] 
29. Chung, C.M.; Lin, M.S.; Hsu, J.T.; Hsiao, J.F.; Chang, S.T.; Pan, K.L.; Lin, C.L.; Lin, Y.S. Effects of statin therapy on cerebrovascular and renal outcomes in patients with predialysis advanced chronic kidney disease and dyslipidemia. J. Clin. Lipidol. 2017, 11, 422-431. [CrossRef] [PubMed]

30. National Health Research Institutes. Taiwan Chronic Kidney Disease Clinical Guidelines; National Health Research Institutes: Taipei, Taiwan, 2015.

31. Cruz-Jentoft, A.J.; Baeyens, J.P.; Bauer, J.M.; Boirie, Y.; Cederholm, T.; Landi, F.; Martin, F.C.; Michel, J.P.; Rolland, Y.; Schneider, S.M.; et al. Sarcopenia: European consensus on definition and diagnosis: Report of the European Working Group on Sarcopenia in Older People. Age Ageing 2010, 39, 412-423. [CrossRef] [PubMed]

32. Mikus, C.R.; Boyle, L.J.; Borengasser, S.J.; Oberlin, D.J.; Naples, S.P.; Fletcher, J.; Meers, G.M.; Ruebel, M.; Laughlin, M.H.; Dellsperger, K.C.; et al. Simvastatin impairs exercise training adaptations. J. Am. Coll. Cardiol. 2013, 62, 709-714. [CrossRef] [PubMed]

33. Panza, G.A.; Taylor, B.A.; Dada, M.R.; Thompson, P.D. Changes in muscle strength in individuals with statin-induced myopathy: A summary of 3 investigations. J. Clin. Lipidol. 2015, 9, 351-356. [CrossRef]

(C) 2020 by the authors. Licensee MDPI, Basel, Switzerland. This article is an open access article distributed under the terms and conditions of the Creative Commons Attribution (CC BY) license (http://creativecommons.org/licenses/by/4.0/). 